\title{
Search for Dark Matters at Accelerators
}

\author{
Giulio Auriemma \\ Universitá degli Studi della Basilicata, Italy and INFN Sezione di Roma,Italy E-mail: \\ giulio.auriemmadcern.ch
}

This talk is a short review of part of the searches of new particles of possible interest for Astrophysics and Cosmology with the different detectors that has been in operation for physics since the year 2010. A consistent fraction of the efforts of the LHC experiment has been devoted to the search for particle and processes not predicted by the Standard Model that could give some input to the understanding of the nature of the dark matter, but with only limited success until now, specially in the search for evidences in favor of the supersymmetry, that are supposed to be able to explain the large WIMP's abundance observed in the Universe. In the paper are discussed the present status of the searches for WIMPs and rare decays after the Run 1 of LHC, in comparison with the direct and indirect searches for the cosmological relic dark matter, but also the discovery of exotic heavy hadronic states (tetra and pentaquarks), that were hypothesized in QCD many years ago, whose existence appears now convincingly proved.

Frontier Research in Astrophysics - II

23-28 May 2016

Mondello (Palermo), Italy 


\section{Introduction}

The occasion of this review of the results of the search of Dark Matter (DM) by the Large Hadron Collider (LHC) of CERN, is the starting, in June 2015, of Run 2 at the world record $\sqrt{s}=13 \mathrm{TeV}$ (that the kinetic energy of a $30 \mathrm{PeV}$ cosmic ray's proton) producing $\simeq 8 \times 10^{13}$ inelastic $p p$ collisions per second.

LHC was approved by CERN Council more than 20 years ago, in December 1994, and saw its "first light" at $\sqrt{s}=900 \mathrm{GeV}$ at the end of September 2008, is at the moment, and will be for many years, the more sensitive instruments suitable for the detection of Beyond Standard Model (BMS) particles. It has been operative for particles search from 30 March 2010 until 10 February 2013, in the so called Run 1, initially at $\sqrt{s}=7 \mathrm{TeV}$ and after at $\sqrt{s}=8 \mathrm{TeV}$.

In the two years shut down following Run I the machine has been prepared to the attainment of a c.m.s. energy close to the goal design of $14 \mathrm{TeV}$. Finally Run 2 has been resumed last month after a technical stop at the same energy and luminosity from March 20. At the present schedule the original design goals requires still a big effort in tests and design, that should be completed for a Run 3, expected not before the year 2021 [1].

A very legitimate question is: Why to search dark matter (DM) at LHC ? After all we do know that the dark matter is extremely abundant in the Universe. Nevertheless the DM in the Earth vicinity has a density of $\simeq 0.3 \mathrm{GeV} / \mathrm{c}^{2}$ per $\mathrm{cm}^{3}$ (see e.g. Ref.[2]), therefore the flux of DM particle's inpinging on any terrestrial detector is

$$
\Phi_{D M}=\frac{\rho_{D M}}{m_{D M}} \times v_{\oplus}=\sim \times 10^{5}\left(\frac{100 \mathrm{GeVc}^{-2}}{m_{D M}}\right)\left(\frac{v_{\oplus}}{270 \mathrm{kms}^{-1}}\right) \mathrm{cm}^{-2} \mathrm{~s}^{-1}
$$

Obviously LHC could discovery DM only if it is produced in high energy $p p$ collisions, but there is a general consensus on the fact that LHC has a good probability of detecting DM, if it has been thermally produced in the Early Universe.

It is discouraging, however, to think how little we really know about DM after so much time from its discovery (For recent reviews see e.g. Ref.s [3, 4]). In fact the first indication of the existence of DM in the Universe has been given by Zwicky in 1933 [5] in one of the first studies on the clusters of galaxies [6]. But our only certainty, until now, is that DM is gravitationally bounds to the galaxies, that means that it couples to the ordinary Standard Model (SM) matter trough gravity (the weakest and the least known interaction at the microscopic level in Nature). The existence of DM is strongly supported by three type of astronomical data:

- Missing mass in galaxies and in groups of them;

- Gravitational lensing of the light of bright galaxies at cosmological distances;

- Small scale anisotropies of the Cosmic Microwave Background Radiation (CMBR).

Excluding controversial claims of detection of DM [7, 8, 9], only upper limits has been given directly or indirectly, from Astrophysical observation until now. We can derive properties of the DM particles only from indirect theoretical evidence that

- Big Bang Nucleosynthesis is incompatible with nuclear Dark Matter [10]; 
- The age of the Universe is incompatible with any weakly interacting neutral particles ("heavy neutrino") with mass smaller than $\sim 3 \mathrm{GeV} / \mathrm{c}^{2}[11]$;

- If DM was pair created after the inflationary reheating, it should have some kind of weak or super-weak interaction with the gauge particles of the SM [12] .

These are, in a nutshell, the basics of the Weakly Interacting Massive Particles (WIMP), the phenomenological model of DM, proposed by Goodman \& Witten about 30 years ago [13]. However we must keep in mind that this model is not the only one proposed for the interpretations of the nature of dark matter, and that some of these are impossible to be tested at accelerators. One obvious example is the MOdification of the Newtonian Dynamics (MOND) on very large astronomical scales, a well known ad hoc solution of the missing mass problem that was invoked by Milgrom [14], and has stimulated the publications of many thousand of refereed theoretical papers until recent [15].

But, with few exceptions, all types of candidates DM particles, such as for example supersymmetric (SUSY) particles, primordial black holes, MACHO's, sterile neutrinos, axions, etc. are expected to be detectable in HEP experiments, with present or near-future technology $[3,16]$.

Traditionally the preferred model for WIMPS, among HEP physicists, has been the SUSY extension of the SM [17], in which the role of WIMP is played by the neutral Less massive SUSY particle (LSP), that areabsolutely stable if the R-parity, being R a quantum number that is 0 for SM particles and \pm 1 for SUSY ones is conserved .

The analysis of the LHC experiments has been done using the two simpler version of the SUSY theory, denominated constrained minimal SUSY model (cMSSM) [18] and phenomenological minimal SUSY model (pMSSM) [19].

Obviously the interest in the SUSY theory, that could a completion (and justification) to SM, is fundamental for HEP physics, if not for Astrophysics. Therefore the exploration of this theory plays a central role in the experimental programme of the LHC. On one side the relatively low mass $\left(\sim 126 \mathrm{GeV} / \mathrm{c}^{2}\right)$ of the Higgs boson, discovered in 2010 at LHC, provides the clue that a vacuum stabilization mechanism, such as that of supersymmetry, should be active at energies $\sim$ few TeV's [20]. But no direct supporting evidence for the theory has been found in Run 1 and the first section part of Run 2 of LHC makes very likely that the typical mass of the SUSY type of particles, coupled to the SM particles (gluino), have masses larger than $1.5 \mathrm{TeV} / \mathrm{c}^{2}$ [21, 22]. Nevertheless, only a very small part of the pMSSM parameters range was explored until now, therefore there is no reason to suspect the SUSY cannot be found by LHC at the design $\sqrt{s}=14 \mathrm{TeV}$, when the luminosity of $\sim 300 \mathrm{fb}^{-1}$ will be reached [23].

In the following $\$ 2$ I discuss the results for a search at LHC of events with large missing transverse energy (MET), that could be possible candidate of WIMP's having masses in a less model-dependent framework than the searches for SUSY, and the comparison of these with the Astrophysical searches. In $\S 3$ and $\S 4$ I will present some recent results of LHC the study of hadronic production and decays that could evidentiate the existence of Beyond the SM (BSM) interactions, that could be related to the nature of DM. The first of the two is dedicated to the search for flavor violating neutral current decay of B mesons, which constitutes a very stringent test of the SM, sensitive to BSM physics in the few TeV energy range [24]. In the second it is discussed the possible 
production of charmed hadrons apparently composed by more than 3 quarks, that could be representative of new class of exotic hadronic DM with mass in the range $\sim 4-10 \mathrm{GeV} / \mathrm{c}^{2}$, that could be not excluded by nucleosinthesis (see e.g. [25] and references therein). Finally in $\$ 5$ a summary of the hot points raised in this talk are listed.

\section{Search for WIMPS at LHC}

Dark Matter is "invisible" not only in the sky but also to collider's detectors if interact only weakly. Nevertheless it can be detected indirectly as Missing Transverse Energy (MET), defined

$$
E_{T}^{m i s s}=-\left|\sum_{i} \vec{p}_{T i}\right|
$$

where $\vec{p}_{T i}$ is the transverse momentum of each particle detected in the event. This method, used for the first time by CERN/UA1 [26] is based on the fact that in a collider all the partons of the two colliding hadrons are with very good approximation co-linear, therefore a large momentum imbalance in the plane transverse to the beam could have been produced only by a massive undetectable particle $\chi_{0}$, with transverse energy :

$$
E_{T}^{m i s s}=\sqrt{M_{\chi}^{2}+p_{T \chi}^{2}} \approx M_{\chi}
$$

This method is applied to events with high $p_{T} \mathrm{X}(\mathrm{X}=\mathrm{jet}, \gamma, W / I Z)$, that have given the trigerr, while events in which the direction of the vectorial sum of missing transverse momentum is in the direction opposite to the $\mathrm{X}$ particle or events with $>1$ high $p_{T}$ jets are vetoed in order to reduce the backgrounds. Remaining backgrounds are (1) $Z^{0} \rightarrow v \bar{v} X, W^{ \pm} \rightarrow \ell^{ \pm} v$ and $Z^{0} \rightarrow \ell^{+} \ell^{-}$where the $\ell$ were missed, (2) QCD backgrounds of multi jets and heavy quarks. Estimate background is evaluated from an accurate MC and validated on exclusive SM processes in the same data. Signal is a deviation $\geq 5 \sigma^{\prime}$ s over the estimated background.

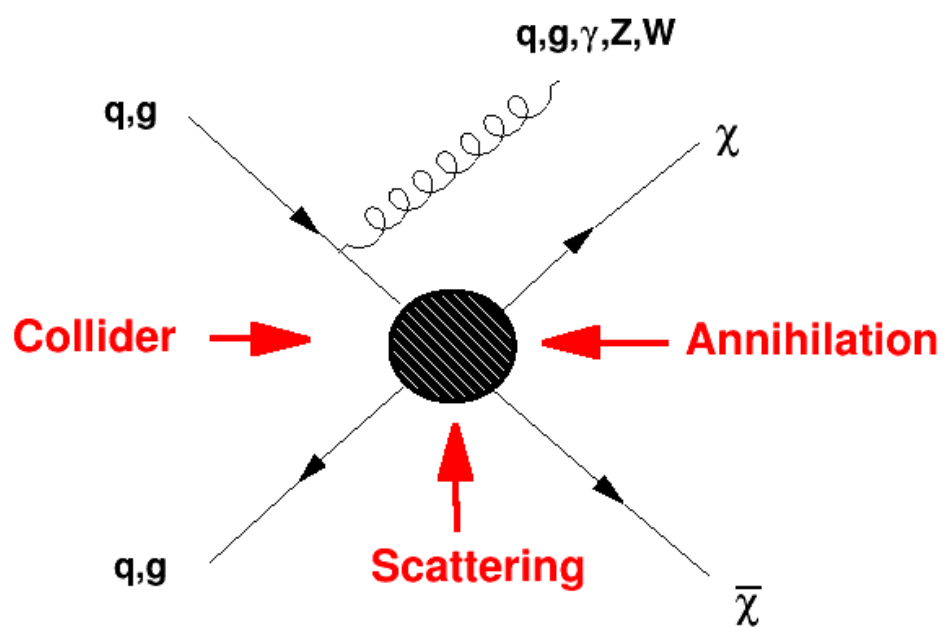

Figure 1: A schematic view of the simplified model of WIMP- hadron interaction. 
Simplified DM Models (SDMM's) [28, 29] can be defined by effective lagrangians, which contains explicitly only a small number of parameters, while most others are integrated out, has been used for the prediction of the MET distribution expected for genuine WIMP's production. These models are clearly less model-dependent of the full SUSY model, and give a simple way to represent different types of searches for new physics. The basic WIMP-Hadron interaction is illustrated schematically in Fig. 1, where the blob mimics the effect of the unknown DM-SM matter interaction. In this simple formulation WIMP's are fermionic Dirac fields that interact via a large mass gauge mediator with the partons of the nuclear matter. In practice the SDMM lagrangian density of the interaction is equal to the sum :

$$
\mathscr{L}_{S D M M}=\sum_{S, V, A, T} \frac{1}{\Lambda_{i}} \mathscr{O}_{i}
$$

extended to all the terms interesting from the point of view of the experimental data. In Table 1 are listed [30] the operators that give the larger contribution to cross sections of production at collider, relic density, annihilation and direct searches of WIMPs.

The SMDM models have four input parameters, the mass of the DM particle $m_{\chi}$, the mass of the mediator $M_{*}$, the coupling of the mediator to the DM particles $g_{\chi}$ and the coupling of the mediator to quarks (or gluons) $g_{q}$. For the latter, as a simplifying assumption, the mediator is assumed to couple to all quark flavors with equal strength.

Table 1: SDMM interaction operators (From [27])

\begin{tabular}{|c|c|c|c|c|}
\hline Name & Initial Sate & Type & $\Lambda_{i}$ & $\mathscr{O}_{i}$ \\
\hline \hline C1 & $q q$ & scalar & $g_{\chi} g_{q} \frac{m_{q}}{M_{*}^{3 / 2}}$ & $\chi^{\dagger} \chi \bar{q} q$ \\
\hline C5 & $g g$ & scalar & $g_{\chi} g_{g} \frac{\alpha_{s}}{4 M_{*}^{2}}$ & $\chi^{\dagger} \chi G_{\mu \nu} G^{\mu \nu}$ \\
\hline D1 & $q q$ & scalar & $g_{\chi} g_{q} \frac{m_{q}}{M_{*}^{2}}$ & $\bar{\chi} \chi q q$ \\
\hline D5 & $q q$ & vector & $g_{\chi} g_{q} \frac{1}{M_{*}^{2}}$ & $\bar{\chi} \chi \gamma^{\mu} \bar{q} \gamma^{\mu} q$ \\
\hline D8 & $q q$ & axial-vector & $g_{\chi} g_{q} \frac{1}{M_{*}^{2}}$ & $\bar{\chi} \chi \gamma^{\mu} \gamma^{5} \bar{q} \gamma^{\mu} \gamma_{\mu} q$ \\
\hline D9 & $q q$ & tensor & $g_{\chi} g_{q} \frac{1}{M_{*}^{2}}$ & $\bar{\chi} \sigma^{\mu v} \chi \bar{q} \sigma_{\mu \nu} q$ \\
\hline D11 & $g g$ & scalar & $g_{\chi} g_{g} \frac{\alpha_{s}}{4 M_{*}^{3 / 2}}$ & $\bar{\chi} \chi G_{\mu \nu} G^{\mu \nu}$ \\
\hline
\end{tabular}

One indication on the mass of the mediator can be obtained from the relic abundance of the DM measured from the small scale fluctuations of CMBR. As shown in Fig. 1 the annihilation channel is the reverse of the production channel. The best estimate of the DM cosmological density from the CMBR is $\Omega_{c} h^{2}=0.1198 \pm 0.0015$ [31]. In the "canonical" approach the annihilation rate that corresponds to an annihilation rate of

$$
\left\langle\sigma_{\chi \bar{\chi}} v\right\rangle_{T_{f}}=2.05 \times 10^{-26} \mathrm{~cm}^{3} \mathrm{~s}^{-1}
$$


at the freeze-out temperature $T_{f} \simeq 25 m_{\chi}$, but only for a DM mass for a mass $m_{\chi}>10 \mathrm{GeV} / \mathrm{c}^{2}$ [32]. In the SDMM the annihilation cross section is

$$
\sigma_{\chi \bar{\chi}}^{a n n} \stackrel{\propto}{\sim} g_{\chi} g_{q} m_{\chi}^{2} / M_{*}^{4}
$$

therefore for $g_{\chi} g_{q} \approx 1$ the two limits at $\sqrt{s}=14 \mathrm{TeV}$ will be for $m_{\chi}=100 \mathrm{GeV} / \mathrm{c}^{2}$ :

$$
1.4 \mathrm{TeV} / c^{2} \leq \frac{M_{*}}{\sqrt{g_{\chi} g_{q}}} \leq 2 \mathrm{TeV} / c^{2}
$$

respectively for over-production and under-production of relic WIMP's, [33]. Range of SDMM parameters that is not excluded by the 2015 Run 2 of LHC [34, 35].

The best U.L. to the cross section of collider production of WIMP's particles have been obtained reached in the $20.3 \mathrm{fb}^{-1}$ data of Run 1 of LHC at $\sqrt{s}=8 \mathrm{TeV}$ is $\sigma_{\text {Jet }+M E T} \leq 730 \mathrm{fb}$ at $95 \%$ C.L. for $E_{T}^{\text {miss }} \geq 250 \mathrm{GeV}$, leading-jet $p_{T} \geq 120 \mathrm{GeV} / \mathrm{c}$ and $|\eta| \leq 2$ [27]. In the SDMM this corresponds (in the average case) to an exclusion region of $M_{*} \lesssim 1 \mathrm{TeV} / \mathrm{c}^{2}$ for $m_{\chi} \leq 100 \mathrm{GeV} / \mathrm{c}^{2}$ which drops to $M_{*} \lesssim 400 \mathrm{GeV} / \mathrm{c}^{2}$ for $m_{\chi} \simeq 1 \mathrm{TeV} / \mathrm{c}^{2}$.

The Fig. 1 WIMP-nucleon cross section is related to the production cross section at collider, as the corresponding $p$-channel process. Therefore the SDMM allows the comparison of the U.L. to the production at LHC $\sigma_{X+M E T}\left(E_{T}^{\text {miss }}\right)$ with the U.L.'s to the direct detection cross section $\sigma_{\chi \mathscr{N}}$ of the direct searches. Fig. 2 shows a compilation of the results of direct searches for DM elastically scattering with nucleons (see [36] and references therein).

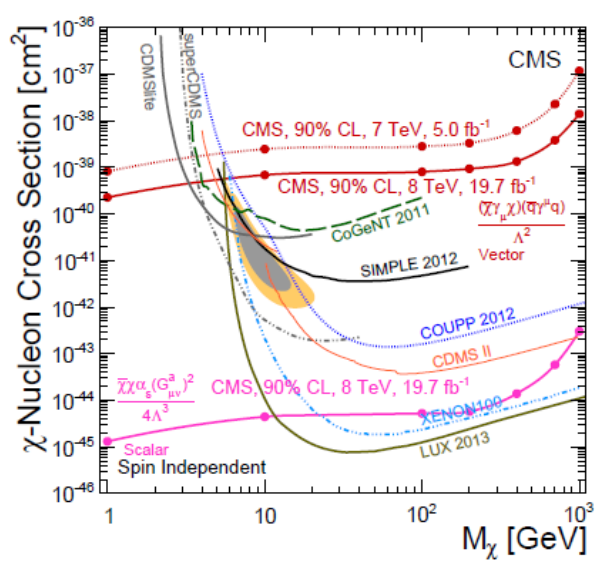

(a)

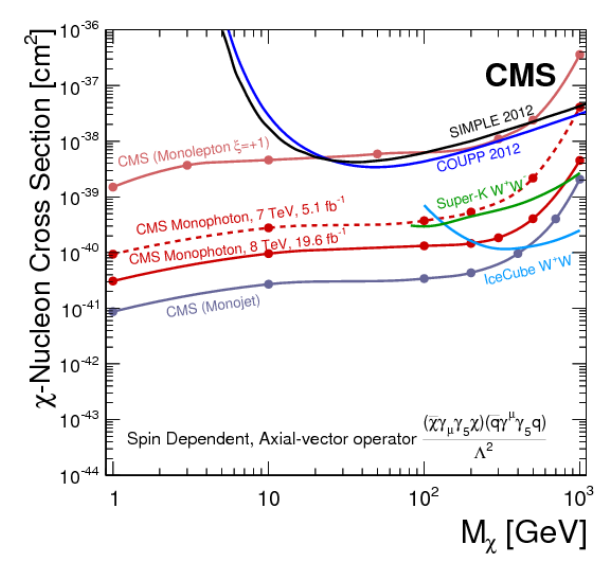

(b)

Figure 2: Upper limits, at $90 \% \mathrm{CL}$, on $\chi$-nucleon elastic scattering cross section as a function of $m_{\chi}$, extrapolated from the SDMM. for spin-independent compared to results from direct searches [36].

The LHC/CMS limits of the SDMM extrapolation WIMP-nucleon spin dependent (SD) scattering for axial-vector interaction type D8 is lower than U.L. cross section of direct searches for $m_{\chi} \leq 250 \mathrm{GeV} / \mathrm{c}^{2}$ : while the limit for spin independent scattering extrapolated from LHC using the SDMM is comparable to that of liquid cryogenic detectors only if the scalar WIMP-gluonic interaction D11 is included. Otherwise the extrapolation based on vector or axial-vector interactions only is at least 5 order of magnitude larger than the direct searches, for $m_{\chi} \geq 10 \mathrm{GeV} / \mathrm{c}^{2}$. Is 
to be noted that, at present, the LHC does not exclude the Dama and CRESST claim in case of axial-vector interaction D8 or similar. Only $\mathrm{n}$ the case of scalar WIMP-gluonic interaction D11 this controversial observation is completely excluded also by the SDMM ${ }^{1}$.

\section{Rare decays}

Rare flavor changing neutral current (FCNC) decays in SM proceed via loop-order diagrams, but new heavy particle, such as WIMP's, competing Feynman diagrams and significantly affect both the branching fractions of the decays and the angular distributions of the final state particles. Studies of rare decays therefore constitute sensitive searches for effects beyond the SM, and furthermore allow to probe the underlying operator structure via global fits . One of the most interesting of this is the old friend $B_{s(d)}^{0} \rightarrow \mu^{+} \mu^{-}$, that has bee observed for the first time by LHCb in 2011 [40] and was later confirmed by CMS [42]. As shown in the Feynman diagrams reported in Fig. 3 the decay (c) is suppressed at tree level by the conservation of the lepton flavor and helicity, but it can be realized through the more complex diagram (d) with very small branching ratio. The most accurate values predicted by the SM are [41]

$$
B R\left(B_{d}^{0} \rightarrow \mu^{ \pm}\right)=(1.06 \pm 0.09) \times 10^{-10}
$$

and

$$
B R\left(B_{s}^{0} \rightarrow \mu^{ \pm}\right)=(3.65 \pm 0.23) \times 10^{-9}
$$
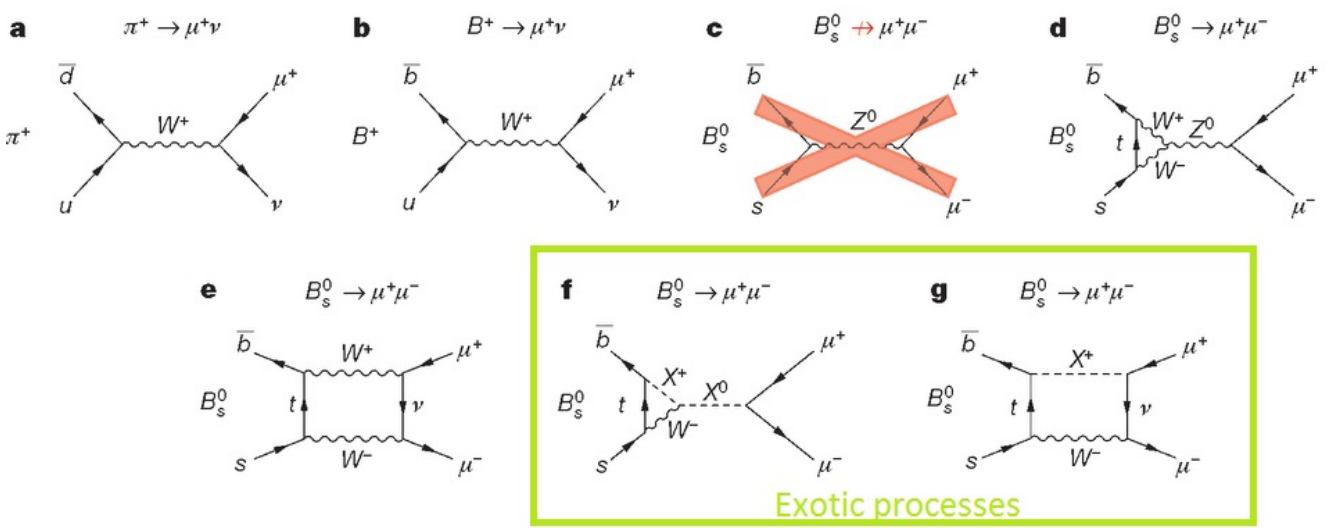

Figure 3: Feynman diagrams of the leptonic decays $B_{(s)}^{0} \rightarrow \ell^{+} \ell^{-}$of the B mesons. The graph (b) is allowed but the (c) is forbidden by flavor conservation. The two penguin and box weak diagrams (d) and (e) originates the rare decay observed, with extremely small branching fraction, The (f) and (g) diagrams are possible only in BSM models.

\footnotetext{
${ }^{1}$ This fact could be a little puzzling, because in the past some theorists haveargued in favor of the possibility that the claimed DAMA and CRESST scintillator's positive detection of DM and the negative outcome of all the liquid-xenon cryogenic detectors could be solved if the scattering of WIMP's could have a different cross section in the two detecting media[37, 38, 39].
} 


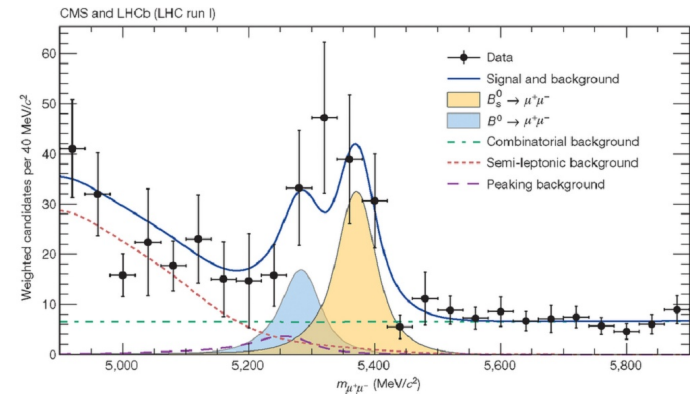

(a)

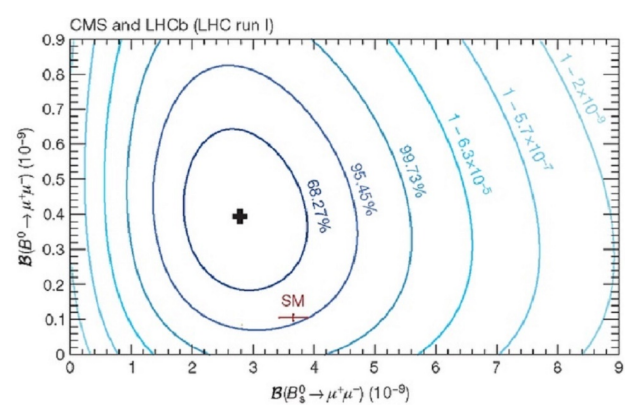

(b)

Figure 4: Panel (a) Combined LHCb-CMS signal [42] after the LHC Run 1. Panel (b) shows $\sim 2 \sigma$ 's tension of the data with the SM predictions

At present, after Run 1, we observe only a not significant tension between our data and the SM that gives $\sim 5-6 \%$ C.L. of the SM hypothesis, that gives a clue to the realization the two BSM diagrams (f) and (g) of Fig. 3. One possibility is the existence of effective operators of the same type of ones invoked for the DM's searches. In fact An exotic mediator with mass $M_{*}$ could produce the decay via the operator $\frac{1}{M_{*}^{2}}\left(\bar{q} \gamma^{\mu} \gamma^{5} q\right)\left(\mu^{ \pm} \gamma^{\mu} \gamma^{5} \mu^{\mp}\right)$, that could give indication of exotic processes, present in the SDMM naturally extended to leptonic fermions. It is worth noticing that the presently available data from Run 1 already exclude $M_{*} \sim \mathrm{TeV} / \mathrm{c}^{2}$ or less.

\section{Multiquarks}

The idea of exotic multiquarks goes back to the early times of QCD [43, 44, 45]. In the SM the only constraint is color charge neutrality and integer electric charge, therefore beside the ordinary mesons and baryons we could have:

- tetraquarks $(q q \overline{q q})$ with the same number of quarks and anti quarks (meson);

- pentaquarks $(q q \bar{q} q \bar{q})$ with triplet of quarks plus a quark-antiquark pair;

- dibaryons $(q q \bar{q} q q \bar{q})$ formed by two triplets of quarks,

Even if there were several unconfirmed claims in the past, as for example the search for the stable dibaryons, predicted by Ref. [45] in a fixed target experiment at the CERN-PS [47], there is now a considerable number of charmed tetraquarks heavy mesons, with masses close to the $D D^{*}$ threshold $\left(\sim 3876.6 \mathrm{MeV} / \mathrm{c}^{2}\right)$, detected in more than one experiment, ${ }^{2}$

- X(3872) Discovered by BELLE in 2003 from the decay $B^{+} \rightarrow\left(J / \psi \pi^{+} \pi^{-}\right) K^{+}$. The quantum numbers of this meson are determined by LHCb to be $J^{P C}=1^{++}$based on angular correlation $B^{+} \rightarrow X(3872) K^{+}$where $X(3872) \rightarrow \pi^{+} \pi^{-} J / \psi$ and $1.0 \mathrm{fb}^{-1}$ of $p p$ collisions collected during Run 1 [50]. This result favors the explanations of the $\mathrm{X}(3872)$ as a tretraquarks state $(c \bar{c} u \bar{u})$

\footnotetext{
${ }^{2}$ Few days later than this conference the LHCb collaboration has officially presented new results [48] about the charmonium spectroscopy, that show the possible existence of three new tetraquarks, denominated $Z(4270), Z(4500)$ and $Z(4700)$, in the proximity of the already known Z(4140), that were, that are not reported correctly in Fig. 5, giving also a full amplitude analysis of their decays [49].
} 
- $\mathrm{Z}(3900)^{ \pm}$discovered as invariant mass peaks of $\left(\pi^{+} \pi^{-} J / \psi\right)$ with $J^{P C}=1^{+-}$that could be $Z_{c}^{+} \equiv(c \bar{c} \bar{d} u)$

- $Z^{0}(3900)$ discovered as invariant mass peaks of $\left(\pi^{0} \pi^{0} J / \psi\right)$ with $J^{P C}=1^{+-}$that could be the $\operatorname{mix}\left(c \bar{c} \frac{\bar{d} u+d \bar{u}}{\sqrt{2}}\right)$

- $\mathrm{Z}(4430)$ Discovered from the decay $B^{0} \rightarrow\left(\psi^{\prime} \pi^{-}\right) K^{+}$a possible $(c \bar{c} d \bar{u})$ state with $J^{p}=1^{+-}$. Is the only candidate at the moment for which an analysis of the angular distribution has been done by LHCb [51] using the Argand's plot methods, which supports the hypothesis of the decay from a real resonance with spin-parity determined unambiguously to be $J^{P C}=1^{+}$.

The pentaquarks was proposed for the interpretation of two heavy hadrons states, respectively as $\Theta^{+}(1540) \equiv\left([u d]^{2} \bar{s}\right)$ and $\Xi^{--}(1840) \equiv\left([d s]^{2} \bar{u}\right)$ ( see e.g. [52] and references therein). But in view of 12 experimental papers completely negative about the $\Theta^{+}(1540)$, the Particle Data Group (PDG) [53] declared in the 2008 reviews that the claimed pentaquark “... was a false alarm” . It is interesting to remark that in their paper of 2003 Jaffe \& Wilczech had given arguments for the existence of heavier pentaquarks including charm and bottom quarks, that could have been stable against the strong decay, impossible to observe at accelerators.

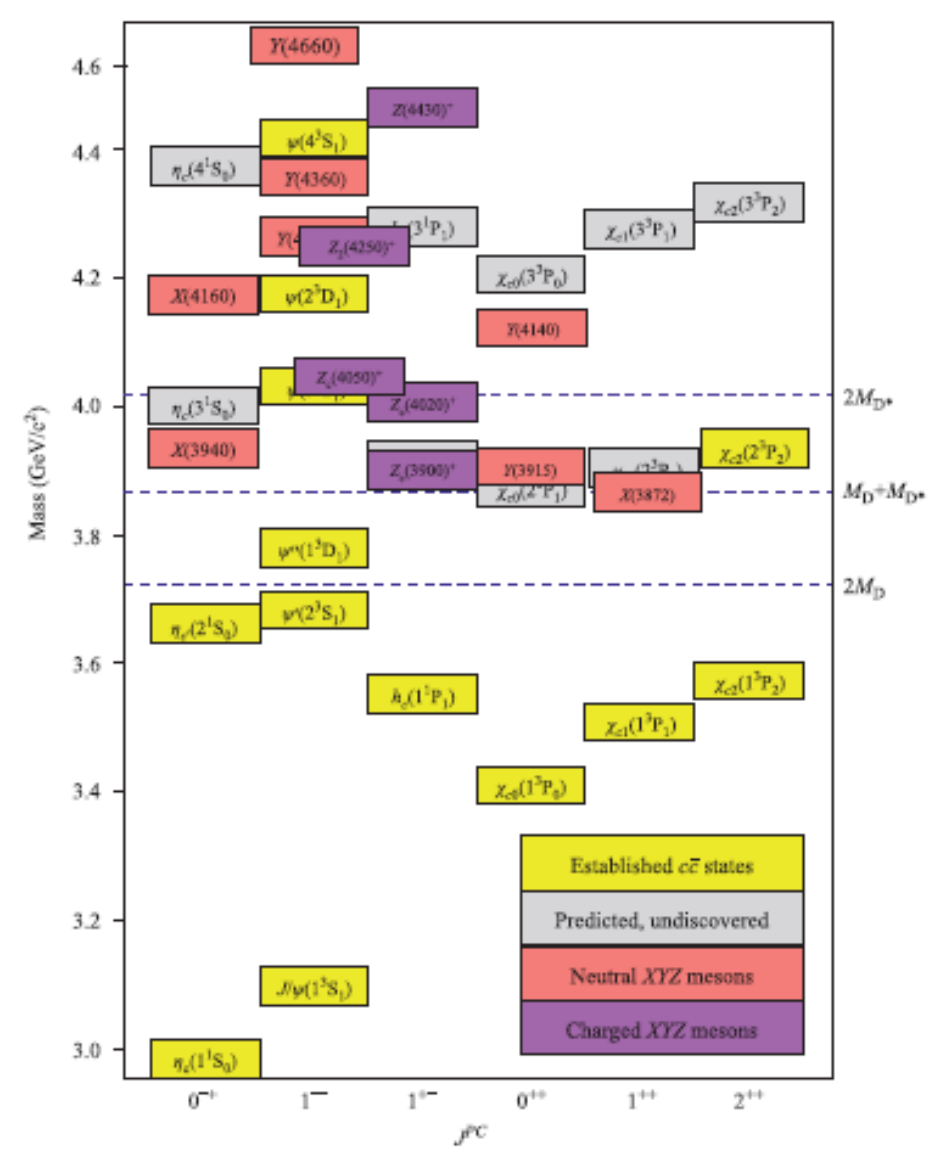

Figure 5: Charmonium spectroscopy (Adapted from[46]) 
Re-analyzing some particular decays of the b-hadrons observed in the Run 1 of LHCb collision data, was found the strange peculiarity in the $\Lambda_{b}^{0} \rightarrow J / \psi p K^{-}$reported in Fig. $6 \mathrm{a}$ and $6 \mathrm{~b}$. The two plots show that in this decay the distribution of the invariant mass both of the $p K^{-}$pairs and of the $J / \psi p$ ones showed a narrow peaks. Two resonance fit the visible excess at $\sim 4.4 \mathrm{GeV} / \mathrm{c}^{-1}$. As shown in Fig. 6(b), the excess can be fitted with two Breit-Wigner shaped resonances:

- $P_{C}^{+}(4380) M=4380 \pm 29 \mathrm{MeV} / \mathrm{c}^{2} \Gamma=205 \pm 86 \mathrm{MeV} \mathrm{J}^{p}=\frac{3}{2}^{-}{ }^{-}(9 \sigma$ 's C.L.)

- $P_{C}^{+}(4450) M=4449,8 \pm 2.5 \mathrm{MeV} / \mathrm{c}^{2} \Gamma=39 \pm 19 \mathrm{MeV} \mathrm{J}^{p}=\frac{5}{2}^{+}(12 \sigma$ 's C.L. $)$

The quark content of this two resonances must be equal to the quarks contained in the decay particles $(c \bar{c} u u d)$.
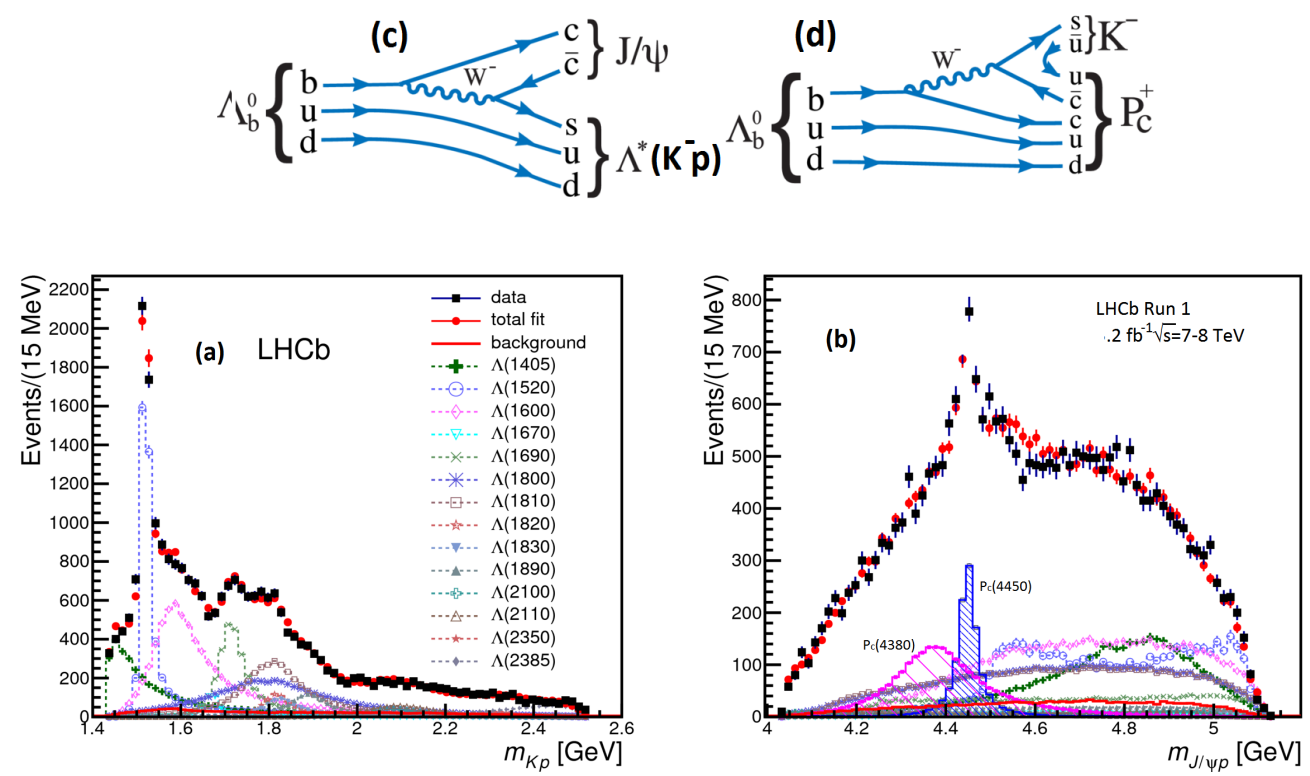

Figure 6: Panel (a) shows the invariant mass distribution of the $K^{-} p$ pairs in the decay $\Lambda_{b}^{0} \rightarrow$ $J / \psi p K^{-}$that shows all the peaks from the well known $\Lambda^{*}$ resonances, dominated by the $\Lambda(1450)$ strange hadron. Panel (b) shows the significant $6.5 \sigma$ unexpected peak at $\approx 4430 \mathrm{MeV} / c^{2}$ of the distribution of the invariant mass of the pairs $J / \psi p$, that is fit by the two resonances $P_{c}^{+}(4450)$ and the less evident $P_{c}^{+}$(4380). In panel (c) is shown the Feynman diagram of the SM decay $\Lambda_{b}^{0} \rightarrow \Lambda_{0}^{*} J / \psi$ followed by the decay $\Lambda_{0}^{*} \rightarrow K^{-} p$ that gives the resonances in panel (a), while panel (d) the possible exotic diagram that drives the production of the pentaquarks state.

In a successive paper the LHCb collaboration has published [54] a new model independent determination of background, inspected for the presence of $J / \psi p$ or $J / \psi K^{-}$contributions, using minimal assumptions about the contributions from the numerous $\Lambda^{*} \rightarrow K^{-} p$ resonances, present at relatively low masses, as shown in Fig. 6(a). It is demonstrated, in this paper, at more than $9 \sigma$ 's that the peak in $J / \psi p$ mass invariant cannot be described as contributions from non-trivial rescatterings production of $J / \psi p$ pairs (as has been argued in Ref.s [55,56]). These model-independent results support well the previously obtained model-dependent evidence for the resonant nature of $P_{c}$ states, observed in the same data sample. 


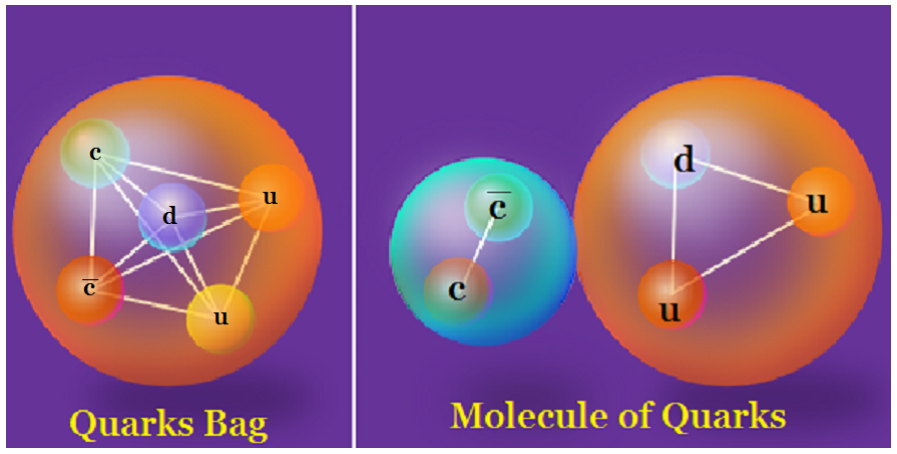

Figure 7: Two possibilities: the "Bag model" or the "Molecular model

Fig. 7 shows the two possible structures for the newly discovered pentaquark. Effectively it could be that both the structure are realized alternated during the lifetime of the resonance. In fact it is known, from nuclear deep inelastic scattering (DIS) experiments [57], that the deuteron appears most of the time as a molecule $(p, n)$, bound by mesonic nuclear forces, while with $\sim 2.2-5.5 \%$ probability appears a six quarks bag [58].

this new type of elementary particles could cosmologically produced only if some of them are stable. It is well known that a proton is stable against the decay $p \not \rightarrow n+e^{+}+v_{e}$ because the mass of proton is $m_{p}<m_{n}$. Similarly the stability of the deuteron against the reaction $D \not \rightarrow p+n$ is given by the fact its mass is $m_{D}=1875.613 \mathrm{MeV} / \mathrm{c}^{2}$ is maller than the sum $m_{p}+m_{n}$ by the $\sim 2.2$ $\mathrm{MeV}$ required for the dissociation. For the same reason we pentaquarks including only one heavy quark, like for example $P_{c}^{0} \equiv\left([u d]^{2} \bar{c}\right)$ (or a fortiori the $P_{b}^{+} \equiv\left([u d]^{2} \bar{b}\right)$ ) are expected to be stable against the decays $P_{c}^{0} \rightarrow p D^{-}$because the invariant mass of charmed meson plus a nucleon are is $m_{p}+m_{D^{-}} \gg 2 m_{u}+2 m_{d}+m_{c}[52,59,60]$. On the contrary the observed pentaquarks $P_{c}^{+}$, witch contains a $c \bar{c}$ pair) are detectable because the strong decay is energetically allowed.

These exotic hadrons could be found in the ultra-dense core of heavy neutron stars or quark stars [62], in DM [63] and/or in cosmic rays [64].

\section{Summary}

The search for SUSY-DM produced $p p$ collisions at LHC is a the moment completely negaative. Comparing the U.L. to the cross section for possible production of SUSY particles accompanied by at least one visible SM particles, in the framework of the pMSSM ( R-parity conserved, LSP is the neutralino $\tilde{\chi}_{0}$, gluino's $\tilde{g}$ or squarks $\tilde{q}$ of $\sim \mathrm{TeV}$ masses produced in $p p$ collisions) gives an extrapolated model dependent L.L. to the SUSY mass scale, that is compatible with a gluino mass $m_{\tilde{g}} \geq 1.4 \mathrm{TeV} / \mathrm{c}^{2}$ or a stop mass $m_{\tilde{t}} \geq 750 \mathrm{GeV} / \mathrm{c}^{2}$ for a neutralino mass $m_{\tilde{\chi}^{0}} \leq 200 \mathrm{GeV} / \mathrm{c}^{2}$, with substantial agreement with the two LHC central spectrometers.

Less model dependent limits on the WIMP-nuclei cross section derived from the LHC experiments are in most of the case very close or better that the limits obtained by the astrophysical searches for cosmological DM either directly on the Earth or in energetic $\gamma$-rays and neutrinos.

Important results have been obtained on the possible existence of multiquark structures, that are not excluded by the SM and speculatively could be an alternative model for Dark Matter. 
At this point of the adventure of LHC one could be tempted to think that the machine is wonderful to explore all the subtleties of the SM but still not at the level of showing its limits . But I hope I have convinced You that this conclusion is premature just at the beginning of Run 2 .

\section{References}

[1] M. Lamont, The LHC machine: Run 2 and beyond, in 50th Rencontres de Moriond, 2015.

[2] M. Weber and W. de Boer, Determination of the local dark matter density in our Galaxy, Astronomy \& Astrophysics 509 (Jan., 2010) A25, [arXiv:0910.4272].

[3] G. Bertone, D. Hooper and J. Silk, Particle dark matter: Evidence, candidates and constraints, Phys. Rept. 405 (2005) 279-390, [hep-ph / 0404175$].$

[4] S. Colafrancesco, Dark Matter in Modern Cosmology, AIP Conf.Proc. 1206 (2010) 5-26, [arXiv: 1004.3869$]$.

[5] F. Zwicky, Die Rotverschiebung von extragalaktischen Nebeln, Helvetica Physica Acta 6 (1933) $110-127$.

[6] G. Bertone and D. Hooper, A History of Dark Matter, Submitted to: Rev. Mod. Phys. (2016), [arXiv:1605.04909].

[7] R. Bernabei et al. (DAMA collaboration), Final model independent result of DAMA/LIBRA-phase1, Eur. Phys. J. C73 (2013) 2648, [arXiv:1308.5109].

[8] R. Aalseth et al. (CoGent collaboration), Search for an Annual Modulation in a P-type Point Contact Germanium Dark Matter Detector, Phys.Rev.Lett. 107 (2011) 141301, [arXiv:1106.0650].

[9] G. Angloher et al., Results on light dark matter particles with a low-threshold CRESST-II detector, Eur. Phys. J. C76 (2016) 25, [arXiv:1509.01515].

[10] A. Dar, Dark matter and big bang nucleosynthesis, Astrophys. J. 449 (1995) 550, [astro-ph/9504082].

[11] E. W. Kolb and M. S. Turner, On the relic abundance of stable neutrinos, Physics Letters B 159 (Sept., 1985) 102-106.

[12] P. A. R. Ade et al. (PLANCK collaboration), Planck 2015 results. XIV. Dark energy and modified gravity, Astronomy \& Astrophysics 594 (Sept., 2016) A14, [arXiv:1502.01590].

[13] M. W. Goodman and E. Witten, Detectability of Certain Dark Matter Candidates, Phys. Rev. D31 (1985) 3059.

[14] M. Milgrom, A modification of the Newtonian dynamics as a possible alternative to the hidden mass hypothesis, Astroph. J. 270 (July, 1983) 365-370.

[15] R. H. Sanders, A historical perspective on modified Newtonian dynamics, Canadian Journal of Physics 93 (Feb., 2015) 126-138, [arXiv:1404.0531].

[16] M. Drees and G. Gerbier, Mini-Review of Dark Matter: 2012, arXiv:1204.2373.

[17] H. Murayama, Physics Beyond the Standard Model and Dark Matter, in Les Houches Summer School - Session 86: Particle Physics and Cosmology: The Fabric of Spacetime Les Houches, France, July 31-August 25, 2006, 2007. arXiv:0704.2276.

[18] G. L. Kane, C. Kolda, L. Roszkowski and J. D. Wells, Study of constrained minimal supersymmetry, Phys. Rev. D 49 (Jun, 1994) 6173-6210. 
[19] C. F. Berger, J. S. Gainer, J. L. Hewett and T. G. Rizzo, Supersymmetry Without Prejudice, JHEP 02 (2009) 023, [arXiv:0812.0980].

[20] G. Altarelli and G. Isidori, Lower limit on the higgs mass in the standard model: An update, Physics Letters B 337 (1994) 141 - 144.

[21] M. Aaboud et al. (ATLAS collaboration), Search for new phenomena in events with a photon and missing transverse momentum in pp collisions at $\sqrt{s}=13 \mathrm{TeV}$ with the ATLAS detector, JHEP 06 (2016) 059, [arXiv:1604.01306].

[22] V. Khachatryan et al.(CMS collaboration), Inclusive search for supersymmetry using razor variables in pp collisions at sqrt(s) =13 TeV, [arXiv:1609.07658].

[23] M. Cahill-Rowley, J. L. Hewett, A. Ismail and T. G. Rizzo, Lessons and prospects from the pMSSM after LHC Run I, Phys. Rev. D91 (2015) 055002, [arXiv: 1407 . 4130].

[24] A. J. Buras, R. Fleischer, J. Girrbach and R. Knegjens, Probing New Physics with the $B_{s}$ to $\mu^{+} \mu^{-}$ Time-Dependent Rate, [arXiv:1303.3820].

[25] S. Sarkar, Big bang nucleosynthesis and physics beyond the standard model, Rept. Prog. Phys. 59 (1996) 1493-1610, [hep-ph/9602260].

[26] G. Arnison et al. (UA1 collaboration), Experimental Observation of Events with Large Missing Transverse Energy Accompanied by a Jet Or a Photon(s) in p anti-p Collisions at $\sqrt{s}=540 \mathrm{GeV}$, Phys. Lett. B139 (1984) 115.

[27] G. Aad et al. (ATLAS collaboration), Search for new phenomena in final states with an energetic jet and large missing transverse momentum in pp collisions at $\sqrt{s}=8 \mathrm{TeV}$ with the ATLAS detector, Eur. Phys. J. C75 (2015) 299, [arXiv: 1502.01518].

[28] D. Alves, Simplified Models for LHC New Physics Searches, J. Phys. G39 (2012) 105005, [arXiv:1105.2838].

[29] J. Alwall, P. Schuster and N. Toro, Simplified Models for a First Characterization of New Physics at the LHC, Phys. Rev. D79 (2009) 075020, [arXiv: 0810 . 3921].

[30] J. Goodman, M. Ibe, A. Rajaraman, W. Shepherd, T. M. Tait et al., Constraints on Dark Matter from Colliders, Phys.Rev. D82 (2010) 116010, [arXiv:1008.1783].

[31] P. C. Ade et al. (PlancK collaboration), Planck 2015 results. XIII. Cosmological parameters, Astronomy \& Astrophys. 594 (2016) A13, [arXiv: 1502 .01589].

[32] G. Steigman, CMB Constraints On The Thermal WIMP Mass And Annihilation Cross Section, Phys. Rev. D91 (2015) 083538, [arXiv: 1502.01884].

[33] G. Busoni, A. De Simone, T. Jacques, E. Morgante and A. Riotto, Making the Most of the Relic Density for Dark Matter Searches at the LHC 14 TeV Run, JCAP 1503 (2015) 022, [arXiv:1410.7409].

[34] M. Aaboud et al. (ATLAS collaboration), Search for new phenomena in final states with an energetic jet and large missing transverse momentum in pp collisions at $\sqrt{s}=13$ ??TeV using the ATLAS detector, Phys. Rev. D94 (2016) 032005, [arXiv: 1604 . 07773].

[35] CMS COLLABORATION collaboration, Search for dark matter production in association with jets, or hadronically decaying $W$ or $Z$ boson at $\sqrt{s}=13 \mathrm{TeV}$, Tech. Rep. CMS-PAS-EXO-16-013, CERN, Geneva, 2016. 
[36] A. de Cosa (ATLAS and CMS collaborations), LHC results for dark matter from ATLAS and CMS, in Proceedings, 12th Conference on the Intersections of Particle and Nuclear Physics (CIPANP 2015): Vail, Colorado, USA, May 19-24, 2015, 2015. [arXiv:1510.01516].

[37] P. Gondolo and G. Gelmini, Compatibility of DAMA dark matter detection with other searches, Phys.Rev. D71 (2005) 123520, [hep-ph/ 0504010 ].

[38] S. Chang, G. D. Kribs, D. Tucker-Smith and N. Weiner, Inelastic Dark Matter in Light of DAMA/LIBRA, [arXiv:0807.2250].

[39] R. Foot, Mirror dark matter and the new DAMA/LIBRA results: A simple explanation for a beautiful experiment, [arXiv:0804.4518].

[40] R. Aaij et al. (LHCB collaboration), First evidence for the decay $B_{s} \rightarrow \mu^{+} \mu^{-}$, Phys.Rev.Lett. 110 (2013) 021801, [arXiv:1211.2674].

[41] C. Bobeth, M. Gorbahn, T. Hermann, M. Misiak, E. Stamou and M. Steinhauser, $B_{s}^{0} \rightarrow \mu^{+} \mu^{-}$in the standard model with reduced theoretical uncertainty, Phys. Rev. Lett. 112 (Mar, 2014) 101801.

[42] V. Khachatryan et al. (LHCB and CMS collaborations), Observation of the rare $B_{s}^{0} \rightarrow \mu^{+} \mu^{-}$decay from the combined analysis of CMS and LHCb data, Nature 522 (2015) 68-72, [arXiv:1411.4413].

[43] Y. Ne'eman, Derivation of strong interactions from a gauge invariance, Nucl. Phys. 26 (1961) 222-229.

[44] M. Gell-Mann, A schematic model of baryons and mesons, Phys. Lett. 8 (1964) 214-215.

[45] R. L. Jaffe, Perhaps a Stable Dihyperon, Phys. Rev. Lett. 38 (1977) 195-198.

[46] S. L. Olsen, A New Hadron Spectroscopy, Front. Phys. 10 (2015) 101401, [arXiv: 1411.7738 ].

[47] C. Pigot et al. (Rome-S ACLAY-VANDERBILt collaboration), Study of the Production of a Strange (S $=-1)$ Dibaryon Production in the Interactions of $\mathrm{K}^{-}$and $\pi^{+}$on Deuterium Below 1.5-GeV/c, Nucl. Phys. B249 (1985) 172-188.

[48] R. Aaij et al. (LHCB collaboration), Observation of $J / \psi \phi$ structures consistent with exotic states from amplitude analysis of $B^{+} \rightarrow J / \psi \phi K^{+}$decays, [arXiv:1606.07895].

[49] R. Aaij et al. (LHCB collaboration), Amplitude analysis of $B^{+} \rightarrow J / \psi \phi K^{+}$decays, [arXiv:1606.07898].

[50] R. Aaij et al. (LHCB collaboration), Determination of the X(3872) meson quantum numbers, Phys. Rev. Lett. 110 (2013) 222001, [arXiv:1302.6269].

[51] R. Aaij et al. (LHCB collaboration), Observation of J/ $\psi$ p Resonances Consistent with Pentaquark States in $\Lambda_{b}^{0} \rightarrow J / \psi K^{-}$p Decays, Phys. Rev. Lett. 115 (2015) 072001, [arXiv: 1507.03414 ].

[52] R. L. Jaffe and F. Wilczek, Diquarks and exotic spectroscopy, Phys. Rev. Lett. 91 (2003) 232003, [hep-ph/0307341].

[53] C. Amsler et al. (Particle Data Group collaboration), Review of Particle Physics, Phys. Lett. B667 (2008) 1-1340.

[54] R. Aaij et al. (LHCB collaboration), Model-independent evidence for J/ $\psi p$ contributions to $\Lambda_{b}^{0} \rightarrow J / \psi p K^{-}$decays, Phys. Rev. Lett. 117 (2016) 082002, [arXiv: 1604.05708 ].

[55] F.-K. Guo, C. Hanhart, Q. Wang and Q. Zhao, Could the near-threshold XYZ states be simply kinematic effects?, Phys. Rev. D91 (2015) 051504, [arXiv: 1411.5584 ]. 
[56] F.-K. Guo, U.-G. Meißner, W. Wang and Z. Yang, How to reveal the exotic nature of the $P_{c}(4450)$, Phys. Rev. D92 (2015) 071502, [arXiv:1507.04950].

[57] L. C. Smith, I. G. Aznauryan, V. Burkert, H. Egiyan and K. Joo, CLAS Results from the First and Second Resonance Regions, in 4th International Workshop on the Physics of Excited Nucleons (NSTAR 2004) Grenoble, France, March 24-27, 2004, pp. 169-177, 2004. DOI.

[58] Y. E. Kim and M. Orlowski, Six quark compound state in deuteron, Phys. Rev. C29 (1984) 2299-2302.

[59] I. W. Stewart, M. E. Wessling and M. B. Wise, Stable heavy pentaquark states, Phys. Lett. B590 (2004) 185-189, [hep-ph/0402076].

[60] S. H. Lee and S. Yasui, Stable multiquark states with heavy quarks in a diquark model, Eur. Phys. J. C64 (2009) 283-295, [arXiv: 0901 .2977].

[61] H. J. Lipkin, Some comments on the possibilities for searches for the pentaquark (anti-c s u u d), Nucl. Phys. Proc. Suppl. 21 (1991) 258-263.

[62] F. Weber, O. Hamil, K. Mimura and R. Negreiros, From Crust to Core: a Brief Review of Quark Matter in Neutron Stars, International Journal of Modern Physics D 19 (2010) 1427-1436.

[63] E. Witten, Cosmic separation of phases, Phys. Rev. D 30 (Jul, 1984) 272-285.

[64] A. de Rujula and S. L. Glashow, Nuclearites - A novel form of cosmic radiation, Nature 312 (Dec., 1984) 734-737. 